\title{
El impacto de la nueva ley de etiquetados de alimentos en la venta de productos en Chile
}

\author{
Valeria Scapini Sánchez* \\ UNIVERSIDAD DE VALPARAISO \\ Cinthya Vergara Silva** \\ UNIVERSIDAD DE CHILE
}

\section{RESUMEN}

Desde la década de los 80 a la fecha la obesidad en Chile se ha triplicado generando problemas económicos en el sector de salud pública y privada. Esto hizo necesario entregar información a los consumidores para que conozcan los ingredientes de cada producto y su valor nutricional. La Ley "Sobre composición nutricional y su publicidad" del ańo 2012, se constituye como parte de una política integral que incluye acciones de promoción, educación, programas de atención primaria y normativa para etiquetar alimentos que alerten con sellos "ALTO EN" en nutrientes relevantes que excedan los límites determinados como riesgosos para la salud. Este estudio busca analizar los efectos de la ley dentro de los primeros 6 meses desde que entra en vigencia tomando como referencia los datos de venta de una empresa del rubro alimenticio que adicionó sellos a sus productos. Los resultados muestran que la Ley sí genera modificaciones en la preferencia de compra de los consumidores y que la cantidad de sellos también es una variable a considerar al momento de comprar un producto.

Palabras Clave: Etiquetado de alimentos, Política nutricional, hábitos alimenticios, comportamiento del consumidor.

\section{Abstract}

Since the 1980s, obesity in Chile has tripled, generating economic problems in the public and private health sector. For this reason is necessary to provide information to consumers about the ingredients of each product and its nutritional value. The Law "On nutritional composition and its advertising" of 2012, has been created as part of a comprehensive policy that includes actions of promotion, education, primary care programs and food labelling regulations that alert through stamps "HIGH IN" in relevant nutrients that exceed the limits determined as risk to health. This study seeks to analyze the effects of the law within the first 6 months using as reference the sales data of a food business that added stamps to its products. The results show that the Law does generate changes in the purchase preference of consumers and that the number of stamps is also a feature considered when a product is purchased.

Keywords: tagged food, nutritional policies, food habits, consumer behavior.

*valeria.scapini@uv.cl

**cvergarasilv@ing.uchile.cl

Las autoras agradecen la colaboración de Víctor Gallardo: Estudiante de Ingeniería Comercial,

Universidad de Valparaíso (victor.gallardo@alumnos.uv.cl) 


\section{INTRODUCCIÓN}

1 bienestar de una sociedad y las condiciones de vida en un país están muy ligados al consumo diario de alimentos y toda la cadena de producción alimenticia. La alimentación de las personas de un país y la calidad de los productos consumidos están estrechamente ligadas a la salud y rendimiento de cada individuo. A nivel mundial es cada vez más importante revisar los hábitos alimenticios debido al aumento de enfermedades ligadas a la ingesta de productos de mala calidad y/o con ingredientes que son perjudiciales para la salud humana. Factores como la ingesta de alimentos poco saludables, bebidas azucaradas, los snacks de alta densidad energética, la "comida rápida”, y el sedentarismo han mostradotener efectos sobre el aumento explosivo de sobrepeso en la población (WCRF, 2009; WHO, 2003).

El sobrepeso y la obesidad se definen como una acumulación anormal o excesiva de grasa que puede ser perjudicial para la salud. Varios estudios muestran que se asocia a problemas de autoestima, depresión e inserción social así como también a enfermedades como la diabetes tipo II, un amplia gama de los cánceres (como cáncer a la próstata y al páncreas), a las enfermedades cardiovasculares, asma, enfermedad de la vesícula biliar, apnea del sueńo, accidente cerebrovascular, problemas respiratorios, enfermedades al hígado y la vesícula biliar, hipertensión y osteoartritis, entre otras.

De acuerdo con cifras de la Organización de Comida y Agricultura Organización de las Naciones Unidas para la Alimentación y la Agricultura FAO (por sus siglas en inglés: Food and AgricultureOrganization) se ha visto un preocupante aumento de la obesidad y sobrepeso. Desde 1980 la obesidad se ha más que doblado en todo el mundo y, al año 2014, se diagnosticaron más de 1.900 millones de adultos de 18 o más años con sobrepeso, de los cuales, más de 600 millones eran obesos y 41 millones de niños menores de cinco años tenían sobrepeso o eran obesos. Esto es términos relativos equivale a que el $39 \%$ de las personas adultas de 18 o más años tenían sobrepeso, y el 13\% eran obesas. De acuerdo con los datos de la 
Encuesta Nacional de Salud 2010 (MINSAL, 2011), en Chile, el $67 \%$ de la población muestra algún grado de sobrepeso, de los cuales un $39,9 \%$ posee un sobrepeso leve, un $25,1 \%$ es obeso y 2,3 es obeso mórbido. Se estima, además, que 1 de cada 11 muertes son producto o están ligadas al sobrepeso.

A su vez, los problemas de sobrepeso han generado en todo el mundo problemas económicos en el sector de la salud pública y en el sector privado (Withrow \& Alter, 2011). Dentro del sector de salud pública se evidencian mayores costos por tasas de admisión, mayor cantidad de días de estancia y costo por persona, mientras que para el sector privado existe directa pérdida de productividad por una mayor tasa de licencias médicas (Korda, y otros, 2015; Fudin, 2013). Los problemas de sobrepesoy de obesidad representan un factor de mayor riesgo en enfermedades no comunicables y, además, es un inductor a mayores costes para el sistema de salud (Kopelman, 2000; Guh, Zhang, Bansback, Amarsi, Birmingham, \& Anis, 2009; Katzmarzyk, Janssen, \& Ardern, 2003; U.S. Department of Health \& Human Service, 2016; Cuadrado C. , 2016). En Chile se estima que las personas obesas generan un $67 \%$ más de gastos en atención de salud en que las personas con un peso normal (Cuadrado C. , 2016).

Se estima que en Chile se gasta alrededor del 0,81\% del PIB y el $5 \%$ del gasto en salud debido a los efectos colaterales de la obesidad, tales como tratamientos médicos, enfermedades subyacentes, pensiones de invalidez, ausentismo laboral, pérdida de productividad y mortalidad prematura. La Asociación Chilena de Seguridad (ACHS) en conjunto con la Universidad Mayor (2016) estimaron que personas con sobrepeso tienen un 31\% más probabilidades de tener un accidente de trabajo mientras que otro estudio realizado por Facultad de Economía y Negocios de la Universidad de Talca estima que un trabajador obeso es 6,34 veces más caro y en términos de licencias presentan una media de 1,37 días de ausencia versus 0,12 días de los no obesos (Tello, 2015).

Chile ocupa el quinto lugar en la tasa de mayor obesidad en el mundo y el primero en Latinoamérica siendo el segundo país con mayor consumo de alimentos ultra procesados en la región 
y el mayor consumidor de bebidas azucaradas del mundo (FAO, 2016).

Como medida para mitigar los preocupantes índices de sobrepeso, el año 2007 por moción de los senadores, Guido Girardi, Carlos Kuschel, Evelyn Matthei, Carlos Ominami, y Mariano Ruíz-Esquide, se presenta el proyecto de ley sobre "Regulación de los alimentos poco saludables". En este marco, el Primer Informe Comisión de Salud (BCN, 2011) siguiendo las orientaciones de la OMS sobre la materia mostró datos para justificar un marco regulatorio especial sobre seguridad alimentaria y alimentación saludable:

"La obesidad es la enfermedad crónica más prevalente en todos los grupos de población chilena (8\% en menores de seis años, $17 \%$ en escolares de Primer Año Básico, 33\% en embarazadas, 28\% en mujeres y $23 \%$ en hombres adultos) y es, a su vez, un factor de riesgo para otras enfermedades, como son las hipercolesterolemias (35\% de los adultos), la hipertensión arterial (33,7\% de los adultos) y la diabetes mellitus tipo 2 (4 a 12\%)."

Posteriormente, se convierte en la Ley 20.606 "Sobre composición nutricional de los alimentos y su publicidad" publicada el ańo $2012(\mathrm{BCN}, 2011)$ y que entró en vigencia el 27 de junio del año 2016 como la "Ley de Etiquetados de Alimentos", que trata sobre composición nutricional de los alimentos y su publicidad, buscando indicar el exceso de nutrientes críticos en aquellos alimentos altos en azúcares, sodio, grasas saturadas y calorías de acuerdo al Reglamento Sanitario de los Alimentos (MINSAL, 1999).

Con todo, la obesidad se considera el problema de salud pública más importante en la actualidad y existe una gran preocupación por cambiar los hábitos alimenticios de la población con miras a mejorar tanto la salud como su calidad de vida. Luego, se espera que tenga como efecto directo una disminución del consumo de productos poco saludables lo que, para las empresas productoras de alimentos procesados, significaría una disminución en las ventas.

Esto ha generado una serie de discusiones y oposición por parte de varias empresas del rubro que no están dispuestas a 
disminuir su margen de ganancia en favor de una mejor calidad de vida para la población chilena.

Dado lo anterior este estudio busca discutir y medir los posibles efectos de la Ley, durante los primeros 6 meses de su puesta en marcha. Analizando por una parte estudios públicos realizados sobre el tema de etiquetado y, por otra, los datos reales de ventas de una empresa del rubro de alimentos procesados que comercializa caramelos, golosinas, galletas, chocolates, galletas, alfajores y cereales con azúcar añadida, helados, entre otros. El principal foco consiste en determinar el impacto que esta nueva ley de etiquetados trae respecto de las ventas de productos y verificar si el consumidor castiga a los productos de acuerdo al número de sellos que tengan sus etiquetas, o si le son indiferentes al momento de comprar. Para determinar este efecto se estimaron una serie de modelos de regresión lineal que permitieron identificar el signo sobre las unidades y monto de venta, $y$ en qué casos es significativo o no.

\section{ETIQUeTado de NUTRICIONAL}

El etiquetado nutricional consiste en la entrega de información en una etiqueta que indique el contenido materias alimenticias que posee un producto alimentario y sus características nutricionales. De manera tal que, los consumidores, sepan fácilmente qué ingredientes posee y su valor nutricional.

Desde la perspectiva de los consumidores, el objetivo primero de la etiqueta nutricional es proporcionar información nutricional que facilite comparar entre productos similares $y$, a partir de ello, los consumidores puedan elegir con conocimiento de causa. De esta manera, podrán evitar productos nocivos o que no respondan a sus preferencias de consumo (González, Romero, Tamer, \& Guerra, 2012). El etiquetado nutricional de los alimentos representa potencialmente una valiosa herramienta para ayudar a los consumidores a tomar decisiones conscientes acerca de su dieta con el fin de mejorar la salud y prevenir enfermedades crónicas (Babio, López, \& Salas-Salvado, 2013; Hennebery \& Armbruster, 2003).

Podemos identificar entonces que el etiquetado nutricional 
pertenece a un sistema que involucra a un grupo de actores que lo controlan, utilizan y norman donde, los actores principales de este sistema son tres: el Estado, como ente regulador y planificador del sistema; la Industria, como abastecedora de productos y de información nutricional en el empaque y; el Consumidor, como receptor de las interacciones y regulaciones de los otros actores.

A nivel mundial el etiquetado nutricional de los alimentos ha ido aumentando su aplicación y complejidad, así como también las exigencias de los consumidores relativas a la información presente en las etiquetas como por ejemplo país de origen, modo de producción, si posee transgénicos, entre otros. Luego, existen tambiénuna serie de elementos estéticos tales como los idiomas de la información; la manera de presentar la información como números, símbolos, categorías, cantidades absolutas, porcentajes, tablas, etc. que son necesarios para llegar adecuadamente a los consumidores. En efecto, la capacidad de comprender las etiquetas puede variar según los países, el estado sanitario de las personas, los objetivos de la sanidad pública, y los patrones alimenticios de los distintos grupos de población dentro de un mismo grupo de consumidores (FAO, 1993; Cowburn \& Stockley, 2005).

\subsection{Ley de etiquetado en Chile}

La Ley de etiquetado en Chile tiene su origen el proyecto de ley sobre "Regulación de los alimentos poco saludables" presentado en el senado el 21 de marzo de 2007 por los senadores, Guido Girardi, Carlos Kuschel, Evelyn Matthei, Carlos Ominami, y Mariano Ruíz-Esquidecon el objetivo de crear un

"[...] marco regulatorio especial sobre seguridad alimentaria $y$ alimentación saludable que recoja los principios y orientaciones internacionales y los haga aplicables en el plano nacional, orientando al consumidor hacia patrones de conducta saludable y advirtiéndola al mismo tiempo sobre los riesgos de consumir alimentos nocivos para su salud.".

Esto permitió que se establecieran las reglas mínimas de la producción, distribución y comercialización de alimentos para ser informadas adecuadamente a los consumidores y, en caso de ser fuente de problemas de salud, que se entregue la información 
claras sobre la calidad y cantidad de lo que está consumiendo (BCN, 2011).

De acuerdo con datos presentados por el Senador don Adolfo Zaldivar distintos estudios avalados por la Organización Mundial de la Salud muestran que al poner en marcha políticas focalizadas en aumentar la actividad física y mejorar la alimentación evita un 90\% de las enfermedades coronarias, 90\% de los infartos y cerca de $70 \%$ por ciento de los accidentes vasculares encefálicos, y casi totalmente, los cuadros de diabetes y el uso de medicamentos para tratarlos, que corresponden además a las primeras y más costosas causas de muerte en Chile y el mundo (BCN, 2011). Dentro de las medidas identificadas como costo efectivas para combatir obesidad se identificaron:

- Etiquetado frontal para una compra informada

- Regular la publicidad dirigida a nińos

- Intervenciones en escuelas

- Consejería en vida sana en atención primaria

- Impuestos alimentos altos en nutrientes críticos

- Promover actividad física

Se promulga entonces, en el año 2012, la ley 20.606 "Sobre composición nutricional y su publicidad", que corresponde a una modificación al Reglamento Sanitario de Alimentos y, se constituye, como parte de una política integral que incluye acciones de promoción, educación, programas de atención primaria, medidas fiscales y otras estrategias de salud pública para combatir la obesidad.

Posteriormente, luego de una serie de discusiones, se promulga el Decreto $13^{\circ}$ de 2015 "Modifica el Decreto Supremo No 977", de 1996, del Ministerio de Salud, que aprueba el "Reglamento Sanitario de los Alimentos"con fecha del 26 de junio de 2015. Este decreto indica que:

Articulo 120 bis: Cuando a un alimento o producto alimenticio se le haya adicionado sodio, azúcares o grasas saturadas, y su contenido supere el valor establecido, el alimento o producto deberá llevar rotulado la o las caracteristicas nutricionales relativas al nutriente adicionado. En el caso de la energía, se deberá rotular su contenido cuando se le haya adicionado azúcares, miel, jarabes, o 
grasas saturadas, y se supere el valor establecido en la referida Tabla".

El reglamento establece límites para el contenido de sodio, azúcar, calorías y grasas saturadas denominados nutrientes críticos, en los alimentos. En caso que los productos los excedan deben llevar un octágono negro con letras blancas, que advierte que el alimento es "ALTO EN" el nutriente que corresponda (ver figura 1) con excepción de los alimentos a los que no se les haya añadido azúcares, sodio o grasa saturada como Cereales con sus derivados en harinas o almidones, extruidos, molidos, triturados, hidrolizados, fideos y productos afines y leguminosas.
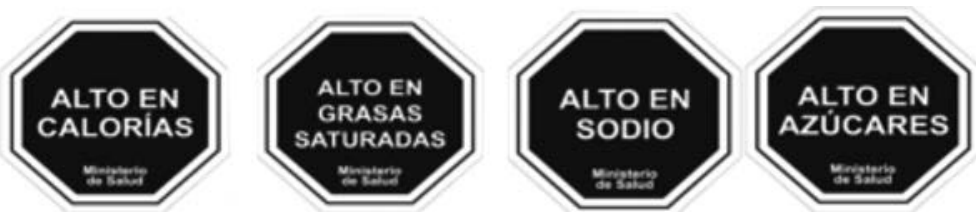

Ilustración 1: Diagrama rotulado sistema de etiquetado chileno. Extraída de Decreto No. 13 oficial de la República de Chile, modificatorio del decreto supremo No. 977, Chile, 2015

Además considera principalmente 3 ejes de acción:

1. Regulación de la publicidad dirigida a menores de 14 años,

2. Implementación de etiquetado de advertencia en los alimentos que superen los límites de nutrientes críticos establecidos para estos efectos y

3. La regulación de la venta de alimentos que superen los límites al interior de las escuelas.

Dada las discusiones ocurridas en el Senado se constató la necesidad de separar algunos aspectos específicos a través de una nueva iniciativa legal que definiera los aspectos relacionados con la publicidad y promoción de los alimentos, creándose así la Ley No 20.869 "Sobre publicidad de Alimentos", publicada en noviembre de 2015, que principalmente legisla sobre la publicidad y promoción de alimentos y define a los menores de edad a las personas con menos de 14 años.

Los alimentos que tenga la etiqueta "ALTO EN" no podrán 
publicitarse a través de canales de difusión orientadas a menores de 14 años, tales como afiches, radio, Internet o programas de televisión cuando capten una audiencia igual o mayor a un $20 \%$ de personas menores de 14 años. También la ley prohíbe utilizar a personajes, figuras infantiles dibujos animados, personas o animales que conciten el interés de los menores de 14 años para publicitar este tipo de productos.

A partir del 26 de junio de 2016 ,y de manera progresiva, todos los alimentos sólidos que posean más de 275 calorías, 400 gramos de sodio, 10 gramos de azúcares totales o 4 gramos de grasas saturadas cada 100 gramos llevaran sellos "ALTO EN". Mientras que, para el caso de los alimentos líquidos, llevaran sello todos aquellos que superen 70 calorías, 100 gramos de sodio, 5 gramos de azúcares totales o 3 gramos de grasas saturadas por cada 100 mililitros (ml).

\subsection{Primeros efectos del etiquetado EN EL MERCAdo CHILENO.}

La ley de etiquetado tuvo un alto consenso parlamentario en relación a su trascendencia e importancia sobre la salud de la población chilena. Sin embargo, no estuvo exenta de oposición sobre todo por parte de la industria de alimentos.

Uno de los principales grupos de representación de la industria, la Asociación de Alimentos y Bebidas de Chile A.G. (AB Chile) y Chilealimentos, definida como:

"Una Asociación de empresas de alimentos en Chile de carácter privado que reune y representa a empresas de alimentos elaborados y compañias de maquinaria, equipos y de servicios vinculadas al procesamiento de los alimentos cuyo objetivo es promover el desarrollo y protección de las actividades que le son comunes a sus asociados y la defensa de sus intereses, en Chile o en el extranjero, ante las autoridades y entidades públicas o privadas" (Chile Alimentos, 2008; Alimentos y Bebidas de Chile A.G. (AB Chile), 1943). donde sus socios fundadores son Andina, Embonor, Coca-Cola, CCU, Carozzi y Nestlé, mostraron gran descontento con la Ley argumentando que el Reglamento aprobado no posee sentido como política pública y no incentiva a la reformulación de 
alimentos debido a las siguientes razones (Leiva, 2014):

1. El Decreto de ley plantea limites que no tienen evidencia cientifica ni sustento de éxito, ya que no han sido aplicado en ningún pais del mundo.

2. La propuesta plantea una estigmatización de alimentos procesados, siendo que ellos representan sólo el 30\% del consumo diario en Chile

3. Desincentivo a la innovación, investigación y desarrollo a la industria Alimentaria.

4. El disco Pare está en desarmonía con Códex Alimentario según sus Normas de Etiquetado Nutricional.

5. Impacto sobre Propiedad intelectual y derechos de marca, especialmente en industrias internacionales.

6. La propuesta genera una barrera técnica al comercio que es innecesaria.

Resaltando como consecuencias que, en resumen, la Ley y el Reglamento no permite discriminar, no educa, atemoriza, desincentiva la innovación de productos, genera impacto sobre propiedad intelectual y derechos de marca incluso, plantean que no respeta acuerdos suscritos por Chile ante la Organización Mundial de Comercio al etiquetar los alimentos que utilizan imágenes infantiles internacionales ya que, la mayoría de las categorías de alimentos, quedarán afectadas muchas en un $100 \%$, llegando a un promedio cercano al $90 \%$ de los alimentos formulados-envasados.

Otros de los puntos más objetados por la industria de alimentos fue el criterio de medición basado en la cantidad nutrientes críticos sobre 100 gramos o $100 \mathrm{ml}$. de cada producto y no en base a la porción recomendada. Sin embargo la Ley fue respaldada con estudios internacionales, actores relevantes del mundo de la salud y centros de estudios que permitieron se aprobara la Ley y el Reglamento a pesar de la molestia de la industria.

Una vez aprobada la Ley se realizaron una serie de evaluaciones con el objetivo de medir su impacto, teniendo en consideración que existen efectos de corto y largo plazo dada la naturaleza de la Ley que busca cambiar costumbres alimenticias arraigadas en 
la sociedad chilena.

De acuerdo a un estudio realizado por el centro de investigación In Situ, iniciado un mes después de entrada en vigencia la ley por solicitud de Chilealimentos, muestra que un $41 \%$ de ellos modificó sus hábitos alimenticios. De los cuales los cambios declarados fueron:1) Disminución en el consumo de productos con discos $(48 \%)$; 2) Sustitución por alimentos similares con menos discos (46\%); 3) Sustitución por alimentos similares sin discos $(26 \%)$; 4) Eliminación de consumo dealimentos con discos (14\%) 5) Otros (3\%). Además un 63\% de los encuestados identificó como relevante o muy relevante la cantidad de sellos en un producto al momento de comprarlo (InSitu, 2016).

Otro estudio de interés realizado por el Centro de Estudios del Retail (CERET) arrojó que al realizar la pregunta sobre la ley de etiquetado ¿Considera que esta nueva información nutricional le ayudó a tomar decisiones de alimentación más saludables? obtuvo como respuestas que un $48,1 \%$ de los encuestados sí le ha ayudado versus un 38,6 que no. Luego, al identificar qué de qué forma afectó el etiquetado en la compra se identificó que un $49 \%$ no modificó su conducta de compra mientras que un 39\% sí lo hizo con acciones como dejar de comprar productos (11\%), Cambio de marca (12\%) y Redujo cantidad (16\%) el resto de los encuestados no sabe o no responde (CERET, 2016).

La encuesta realizada por CADEM (2016), arrojó, en términos de opinión, que el $77 \%$ de los encuestados cree que la ley va "en la dirección correcta porque mejorarán los hábitos alimenticios de las personas" mientras que sólo un $17 \%$ cree que va "en la dirección equivocada porque es un exceso de regulación a las empresas". Además, dentro de los que han visto el etiquetado (82\% de la muestra) un $51 \%$ cree que "tendrá un impacto positivo porque mejorará la calidad de vida de las personas" y un $46 \%$ indica que ha dejado de comprar algún producto de tu consumo habitual por ver que tiene un disco pare negro que dice "ALTO EN" versus un 52\% que no. Por último un 31\% de este grupo muestra lealtad a la marca y "seguiría comprando porque es su marca preferida" mientras un 66\% "eliminaría de su consumo el producto de forma definitiva" (28\%) o "se cambiaría 
de marca a una que no tenga disco pare" (38\%).

Por su parte el Ministerio de Salud (MINSAL, 2017) advirtió que los cambios que se esperan con la nueva ley no serán instantáneos indicando que el efecto en el estado nutricional de la población, gracias a un cambio de conducto en sus hábitos alimenticios, podrá evaluarse en el largo plazo en alrededor 10 años.

En su primera evaluación de la ley utilizó el estudio de Percepción Ciudadana realizado en conjunto por Instituto de Comunicación e Imagen (ICEI) y consultora de evaluación social Demoscópica. Este estudio indica que el 92,4\% de los encuestados evalúa como "bien" o "muy bien" la obligación de etiquetar con sellos de advertencia los alimentos "ALTO EN". En relación a las restricciones de venta de alimentos "ALTO EN", el 91,3\% de los entrevistados consideró que está "bien" o "muy bien" prohibir su venta en establecimientos escolares. Y, en relación a, la prohibición de publicidad dirigida a niños y niñas menores de 14 un $74,5 \%$ estima como "bien" o "muy bien".

Seleccionando dentro de la muestra a aquellos que comparan la cantidad de sellos,

"Un $91,6 \%$ de los consumidores/as considera que la existencia de sellos en los alimentos envasados influye en alguna medida en su decisión de compra, y sólo un $8,4 \%$ señala que los sellos no influyen y que compran igual"

de los primeros, un $67,8 \%$ modifica su decisión de compra hacia alimentos con menos sellos, un 14,1\% manifiesta que compra menos alimentos de los que hubiese comprado si no tuvieran sellos y un 9,7\% no compra alimentos con sellos. Los que declaran no ser afectados por los sellos en su decisión de compra corresponden al 8,4\% de la submuestra.

Adicionalmente, respecto a los efectos en la industria, Ministerio de Salud (2017) identificó a diciembre de 2016 que un $64,4 \%$ de los establecimientos cumplía con las indicaciones de la Ley. Por su parte y de acuerdo a datos entregados por la Sociedad de Fomento Fabril (SOFOFA) un 17,7\% de los productos de alimentos realizaron cambios en la composición de algunos alimentos (MINSAL, 2017). 
Es así como, luego de 6 meses de implementada la Ley, vemos que la población chilena muestra interés por ella incluso disposición a cambiar su comportamiento de compra frente a los sellos. El MINSAL identifica a esta ley como una política pública pionera a nivel mundial en materia de alimentación saludable sin dejar de lado los desafíos permanentes de mejora, como por ejemplo incluir mensajes en braille, la evaluación de sus efectos para combatir los problemas de sobrepeso y el fomento de una alimentación saludable.

\section{Efecto de sellos "AlTO EN" eN UNA EMpresa De} ALIMENTOS.

El principal foco de este artículo es cuantificar con técnicas econométricas el efecto real de la ley de etiquetado,utilizando los datos de venta de una empresa de alimentos chilena.

La empresa bajo estudio posee 400 productos para venta en supermercados, los cuales fueron divididos en 4 grupos de acuerdo a la cantidad de sellos. La empresa posee productos con $0,1,2$ y 3 sellos. De cada grupo se seleccionaron 3 productos más un producto sin sellos que corresponde a un producto sin azúcar añadida (único producto que vende la empresa de cero sellos). La selección de los productos fue de acuerdo a los que representaban mayor venta, que no tuvieran sesgo estacional y que se mantuvieran disponibles a lo largo del periodo bajo estudio.

La base de datos corresponde a una serie de tiempo con 10.861 registros de ventas diarias al detalle dentro de la cadena de supermercado que presenta la mayor participación de las ventas en la Región Metropolitana. Cada registro posee costos, unidades vendidas, número de sellos del envase, ventas, la fecha de la implementación de la Ley y fecha. De los registros 907 días corresponden a ventas antes del comienzo de ley y 179 días de ventas tras el comienzo de la ley.

Tabla 1: Descripción de la base de datos

\begin{tabular}{|l|l|}
\hline \multicolumn{2}{|c|}{ Caracterización de la Base de Datos } \\
\hline Observaciones & 10.861 \\
\hline Productos & 10 \\
\hline Periodo observado & Enero de 2014 a Diciembre 2016 \\
\hline
\end{tabular}




\section{I Metodología}

Para realizar el análisis primero fue necesario limpiar la base datos recibida. Para ello, se quitaron aquellos registros donde existía alguno de los campos en blanco (estrategia de eliminación listwise) obteniendo un nuevo conjunto de datos (ver tabla 2) con 10.678 registros válidos equivalentes el $99 \%$ de la base original.

Tabla 2: Resumen de datos utilizados para el ajuste de modelos

\begin{tabular}{|l|c|r|}
\hline \multicolumn{1}{|c|}{ ID Producto } & \#Sellos & Total Reg. \\
\hline Producto 1 & 2 & 1074 \\
\hline Producto 2 & 0 & 1002 \\
\hline Producto 3 & 2 & 1073 \\
\hline Producto 4 & 3 & 1077 \\
\hline Producto 5 & 1 & 1075 \\
\hline Producto 6 & 1 & 1075 \\
\hline Producto 7 & 1 & 1077 \\
\hline Producto 8 & 3 & 1075 \\
\hline Producto 9 & 2 & 1075 \\
\hline Producto 10 & 3 & 1075 \\
\hline Total general & - & $\mathbf{1 0 6 7 8}$ \\
\hline
\end{tabular}

Los datos fueron procesados con el lenguaje $\mathrm{R}$ utilizando la interfaz gráfica R-Studio. La técnica para evaluar si la ley tuvo incidencia corresponde a modelos de regresión lineal (Wilkinson \& Rogers, 1973; Chambers, 1992) que permite identificar si las variables se ajustan a los resultados de la empresa analizando los coeficientes de la regresión.

En este estudio se estimaron distintos modelos de regresión lineal de Mínimos Cuadrados Ordinarios (MCO) que consideran como variable explicada al logaritmo de las ventas (unidades y monto) para suavizar la curva, y se utilizan como variables explicativas los sellos, la entrada en vigencia de la Ley y los productos. 


\subsection{AnÁlisis de DATOS y estimación de modelos}

El promedio de ventas anual por año alcanza a $\$ 8.694 .864 .317$ unidades monterías para el año 2014, \$9.074.476.731 para el año $2015 \$ 9.969 .820 .305$ para el año 2016. Es posible ver una pequeña variación de ventas estacional y un alza creciente sostenida para cada ańo. En términos de unidades vendidas el comportamiento es menos homogéneo que el de las ventas, sin embargo, en promedio no hay grandes saltos de valor manteniéndose en torno a 7.365 unidades promedio vendidas por mes.

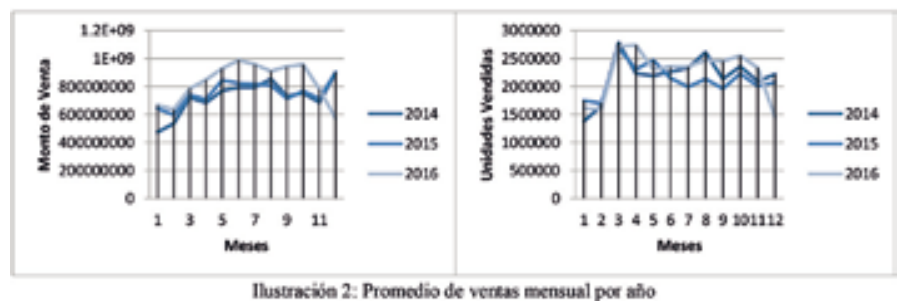

Las ventas anuales de los productos de la muestra se mantienen dentro del mismo rango con excepción de los productos 4 y 10 que presentan un alza anormal el ańo 2016.

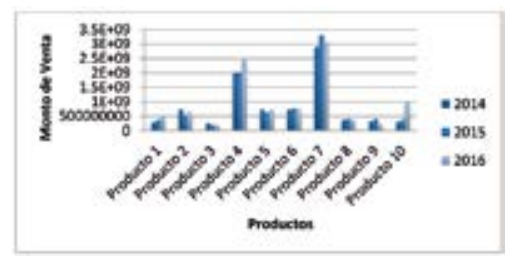

Ilustración 3: Venta promedio por producto

El primer modelo desarrollado corresponde a una evaluación simple de la variable ley sobre las ventas y las unidades. El resultado indica que la ley sí explica en parte los cambios de comportamiento sobre las ventas y que tanto para las unidades como para los montos afecta de manera positiva, y en mayor proporción, a las unidades. Este simple análisis, sin embargo, puede estar afectado por el crecimiento sostenido de las ventas de los productos seleccionados, lo que explicaría el signo del 
coeficiente por lo que no permite por sí sólo generar conclusiones respecto a los efectos de la ley.

Tabla 3: Efecto 1ey sobre ventas y unidades

\begin{tabular}{|c|c|c|c|c|}
\hline \multirow[t]{2}{*}{$\mathbf{Y} \sim \mathbf{X}$} & \multicolumn{2}{|c|}{ Ventas (monto) } & \multicolumn{2}{|c|}{ Unidades } \\
\hline & Estimate & $\operatorname{Pr}(>|t|)$ & Estimate & $\operatorname{Pr}(>|t|)$ \\
\hline (Intercept) & 14.2341047 & $<2 \mathrm{e}-16^{* * *}$ & 8,256 & $<2 \mathrm{e}-16^{* * *}$ \\
\hline \multirow[t]{2}{*}{ Ley } & 0.1158168 & $1.19 \mathrm{e}-05^{* * *}$ & 0.13018 & $4 \mathrm{e}-05^{* * *}$ \\
\hline & \multicolumn{4}{|c|}{ 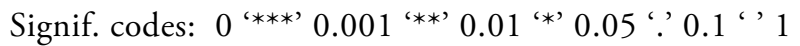 } \\
\hline
\end{tabular}

Luego otros dos modelos para evaluar el efecto de la ley y la cantidad de sellos sobre las ventas y las unidades vendidas de acuerdo a las siguientes regresiones:

$$
\begin{gathered}
\log (\text { ventas })=l e y+\text { sellos }+ \text { ley }{ }^{*} \text { sellos } \\
\log (\text { unidades })=l e y+\text { sellos }+ \text { ley } * \text { sellos }
\end{gathered}
$$

Los resultados obtenidos (ver tabla 4) indican que para todo el periodo la ley sí posee un efecto significativo tanto sobre las ventas como las unidades, en ambos caso el efecto es negativos y existe un mayor impacto sobre los montos de venta que sobre las unidades vendidas. En relación a los sellos también se evidencia que existe un efecto negativo significativo en relación a las ventas $y$ unidades vendidas con menor impacto sobre las unidades que sobre las ventas. unidades

\begin{tabular}{|c|c|c|c|c|}
\hline & \multicolumn{2}{|c|}{ Ventas (monto) } & \multicolumn{2}{|l|}{ Unidades } \\
\hline & Estimate & $\operatorname{Pr}(>|\mathrm{t}|)$ & Estimate & $\operatorname{Pr}(>|t|)$ \\
\hline (Intercept) & 14,592 & $<2,00 \mathrm{E}-16^{* * *}$ & 8,367 & $<2 \mathrm{e}-16^{* * *}$ \\
\hline Ley & $-0,185$ & $0.000705^{* * *}$ & $-0,058$ & 0.3822 \\
\hline Sellos & $-0,1972$ & $<2,00 \mathrm{E}-16^{* * *}$ & $-0,061$ & $4.42 \mathrm{e}-06^{* * *}$ \\
\hline \multirow[t]{2}{*}{ ley:sellos } & 0,1655 & $5.25 \mathrm{e}-10^{* * *}$ & 0,104 & $0.0013^{* *}$ \\
\hline & \multicolumn{4}{|c|}{ 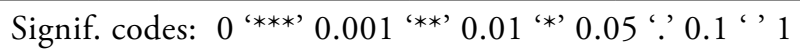 } \\
\hline
\end{tabular}

Tabla 4: Efecto conjunto 1ey y sellos sobre ventas y

Luego, para entender mejor el problema, se realizó un 
modelo para analizar la relación entre las ventas y los productos e identificar qué comportamiento muestran los productos durante todo el periodo, antes de la ley y después de la ley de acuerdo al modelo: ventas_ $i=\beta_{i} *$ productos $i$ con i productos bajo análisis. Para evitar el problema de perfecta colinealidad entre las variables, la categoría omitida Producto 1 es utilizada como base. Los resultados obtenidos se muestran en la tabla 5 .

Tabla 5: Efecto conjunto 1ey y productos sobre ventas y unidades

\begin{tabular}{|c|c|c|c|c|c|}
\hline \multirow[b]{2}{*}{ Variable } & \multirow[b]{2}{*}{$\begin{array}{l}\text { NoSellos } \\
\text { (Ref.) }\end{array}$} & \multicolumn{2}{|c|}{ Ventas (monto) } & \multicolumn{2}{|c|}{ Unidades } \\
\hline & & Estimate & $\operatorname{Pr}(>|t|)$ & Estimate & $\operatorname{Pr}(>|t|)$ \\
\hline Intercepto & - & 1,367 & $<2,00 \mathrm{E}-16^{* * *}$ & 7,827 & $<2,00 \mathrm{E}-16^{* * *}$ \\
\hline Prod. 10 & - & 0,127 & $1.50 \mathrm{e}-06^{* * *}$ & $-0,738$ & $<2,00 \mathrm{E}-16^{* * *}$ \\
\hline Prod. 2 & - & 0,623 & $<2,00 \mathrm{E}-16^{* * *}$ & $-0,709$ & $<2,00 \mathrm{E}-16^{* * *}$ \\
\hline Prod. 3 & - & $-0,395$ & $<2,00 \mathrm{E}-16^{* * *}$ & $-0,355$ & $<2,00 \mathrm{E}-16^{* * *}$ \\
\hline Prod. 4 & - & 1,791 & $<2,00 \mathrm{E}-16^{* * *}$ & 1,303 & $<2,00 \mathrm{E}-16^{* * *}$ \\
\hline Prod. 5 & - & 0,627 & $<2,00 \mathrm{E}-16^{* * *}$ & 1,929 & $<2,00 \mathrm{E}-16^{* * *}$ \\
\hline Prod. 6 & - & 0,773 & $<2,00 \mathrm{E}-16^{* * *}$ & 1,041 & $<2,00 \mathrm{E}-16^{* * *}$ \\
\hline Prod. 7 & - & 2,169 & $<2,00 \mathrm{E}-16^{* * *}$ & 1,444 & $<2,00 \mathrm{E}-16^{* * *}$ \\
\hline Prod. 8 & - & $-0,081$ & $0.002036^{* *}$ & 1,189 & $<2,00 \mathrm{E}-16^{* * *}$ \\
\hline Prod. 9 & - & 0,000 & 0.994767 & $-0,910$ & $<2,00 \mathrm{E}-16^{* * *}$ \\
\hline Ley & - & 0,046 & $<2,00 \mathrm{E}-16^{* * *}$ & 0,589 & $<2,00 \mathrm{E}-16^{* * *}$ \\
\hline Prod. 10:Ley & 3 & 0,508 & $6.36 \mathrm{e}-15^{* * *}$ & 0,537 & $1.72 \mathrm{e}-14^{* * *}$ \\
\hline Prod. 2:Ley & 0 & $-0,462$ & $1.53 \mathrm{e}-12^{* * *}$ & 0,016 & 0.82094 \\
\hline Prod. 3:Ley & 2 & $-0,882$ & $<2,00 \mathrm{E}-16^{* * *}$ & $-0,902$ & $<2,00 \mathrm{E}-16^{* * *}$ \\
\hline Prod. 4:Ley & 3 & $-0,239$ & $0.000236^{* * *}$ & $-0,220$ & $0.00166^{* *}$ \\
\hline Prod. 5:Ley & 1 & $-0,527$ & $6.25 e-16^{* * *}$ & $-0,540$ & $1.21 \mathrm{e}-14^{* * *}$ \\
\hline Prod. 6:Ley & 1 & $-0,568$ & $<2,00 \mathrm{E}-16^{* * *}$ & $-0,577$ & $<2,00 \mathrm{E}-16^{* * *}$ \\
\hline Prod. 7:Ley & 1 & $-0,513$ & $3.15 \mathrm{e}-15^{* * *}$ & $-0,520$ & $1.07 \mathrm{e}-13^{* * *}$ \\
\hline Prod. 8:Ley & 3 & $-0,261$ & $5.84 \mathrm{e}-05^{* * *}$ & $-0,208$ & $0.00293^{* *}$ \\
\hline Prod. 9:Ley & 2 & $-2,025$ & $<2,00 \mathrm{E}-16^{* * *}$ & $-2,078$ & $<2,00 \mathrm{E}-16^{* * *}$ \\
\hline & & \multicolumn{2}{|c|}{ Multiple R-squared: 0.698} & \multicolumn{2}{|c|}{ Multiple R-squared: 0.7571} \\
\hline & & \multicolumn{2}{|c|}{ Adjusted R-squared: 0.6975} & \multicolumn{2}{|c|}{ Adjusted R-squared: 0.7566} \\
\hline & & \multicolumn{4}{|c|}{ Signif. codes: $0^{\text {‘***’ }} 0.001^{\text {‘**’ }} 0.01^{\text {‘*’ }} 0.05^{\prime} ?^{\prime} 0.1^{\prime \prime}{ }^{\prime} 1$} \\
\hline
\end{tabular}


De acuerdo a estos resultados, se puede ver que los productos 3, 8 y 9, antes de que comenzara a regir la Ley, ya presentaban una disminución en sus ventas (tendencia de compra a la baja). En particular los productos 3 y 9 muestran tendencia a la baja tanto en ventas como en unidades y es posible ver que la Ley aumenta esta tendencia a la baja en el consumo. El producto 10 es de especial interés ya que es un producto con 3 sellos que muestra que, tras la aprobación de la ley, aumentó su venta considerablemente, cambiando incluso la tendencia de venta que mostraba anteriormente. El producto 2 (que no posee sellos) muestra que la ley afecta positivamente las unidades vendidas pero no el monto de las ventas. Finalmente, los productos 3, 4, $5,6,7,8$ y 9 muestran que la ley tuvo un efecto negativo tanto en las ventas como en las unidades vendidas.

Por otro lado, analizando sólo el periodo con la Ley en vigencia, se identifica que los productos en los que la ley afectó negativamente fueron aquellos con dos sellos (ver tabla 6).

Tabla 6: Efecto por número de sellos sobre ventas y unidades tras aprobación de la ley

\begin{tabular}{|c|c|c|c|c|}
\hline & \multicolumn{2}{|l|}{ Ventas } & \multicolumn{2}{|c|}{ Unidades } \\
\hline & Estimate & $\operatorname{Pr}(>|t|)$ & Estimate & $\operatorname{Pr}(>|t|)$ \\
\hline Intercept & 14,44 & $<2 \mathrm{e}-16^{* * *}$ & 7,72 & $<2 \mathrm{e}-16^{* * *}$ \\
\hline Sello $=1$ & 0,49 & $7.71 \mathrm{e}-11^{* * *}$ & 1,62 & $<2 \mathrm{e}-16^{* * *}$ \\
\hline Sello $=2$ & $-1,26$ & $<2 \mathrm{e}-16^{* * *}$ & $-0,72$ & $<2 \mathrm{e}-16^{* * *}$ \\
\hline \multirow[t]{4}{*}{ Sello $=3$} & 0,45 & $2.02 \mathrm{e}-09^{* * *}$ & 1,31 & $<2 \mathrm{e}-16^{* * *}$ \\
\hline & \multicolumn{2}{|c|}{ Multiple R-squared: 0.4451} & \multicolumn{2}{|c|}{ Multiple R-squared: 0.5526} \\
\hline & \multicolumn{2}{|c|}{ Adjusted R-squared: 0.4441} & \multicolumn{2}{|c|}{ Adjusted R-squared: 0.5519} \\
\hline & \multicolumn{4}{|c|}{ 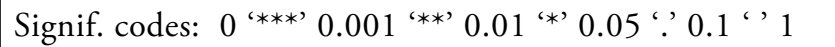 } \\
\hline
\end{tabular}

\subsection{AnÁlisis de modelos y discusión de Resultados}

Los modelos obtenidos muestran que la ley efectivamente tiene incidencia sobre las ventas, tanto en el monto como en las unidades, y es posible considerarla como una variable explicativa 
al cambio de tendencias que se genera dentro del periodo bajo análisis. Las ventas de productos con 1 sello aumentan levemente en relación a los productos que no presentan sellos, mientras que los productos que tienen 2 sellos muestran una disminución del promedio de sus ventas en relación a los productos sin sellos, y por último, los productos de 3 sellos presentan un aumento en el valor esperado de sus ventas.

Los productos en general muestran un cambio de patrón en su serie de ventas con una disminución importante de ventas en los meses noviembre y diciembre de 2016. Dados los resultados, pareciera que los productos con 3 sellos muestran tener un público fidelizado y/o de alta dependencia al producto, tal vez sin productos sustitutos que permitan a las personas buscar una opción más saludable o que el sustituto existente también posea 3 o más sellos con un coste igual o mayor. Los productos con 2 sellos muestran ser los más afectados por la ley y, además, los únicos afectados de manera negativa. Esto podría ser causa de una baja dependencia, lealtad, o que poseen mayor alternativas de sustitución. El producto sin sellos no muestra gran variación en el monto de ventas pero sí en las unidades vendidas, sin embargo, esta leve tendencia pareciera ser resultado de una tasa de crecimiento constante, propia del producto, independiente de la entrada en vigencia de la Ley.

Dentro de los datos facilitados por la empresa no se indica qué tipo de promociones en góndola se realizaron durante el periodo, ni tampoco si hubo cambios en los avisos publicitarios que pudiesen haber afectado el comportamiento del consumidor para mitigar los efectos de la ley. Con ello, los modelos arrojan resultados interesantes pero no totalmente concluyentes respecto a los efectos cuantitativos.

Para complementar estos resultados, es importante considerar la situación de mercado vinculada al mundo alimentario. Por ejemplo, las estrategias utilizadas por las marcas en su etiquetado para confundir a los consumidores con palabras como "natural", "artesanal", "light", "0\% de" o destacar ingredientes que vienen 
en dosis mínima para hacerlos parecer saludables o naturales (López, 2017).

Por otro lado, los consumidores están cada vez mejor informados y muestran a nivel mundial que ha aumentado la educación en relación a los ingredientes contenidos en los alimentos procesados con preocupación también por la forma en que se producen inclusive si el desarrollo del producto tiene en cuenta el bien común de la población. En la misma línea los consumidores han dejado de considerar a las grasas como algo negativo en sí mismo (MINTEL, 2017), entendiendo además que muchas veces los productos procesados cambian grasas por azúcar sin generar ningún beneficio al evitarlas.

Las tendencias mundiales de alimentación muestran un gran crecimiento del interés por la comida saludable, que entregue energía, que no dañe el medio ambiente ni a los animales, con ingredientes más tradicionales y naturales, sin procesamiento industrial (Google, 2016; MINTEL, 2017).

Fenómenos como el anterior podrían explicar la tendencia de venta del producto sin sellos de la empresa, ya que no cambió su comportamiento histórico tras ser aprobada la ley. Los otros productos muestran cambios en mayor o menor medida en su tendencia histórica tras la aprobación de la ley, lo que dada la ventana de análisis podríamos indicar que es un cambio rápido de conducta de los consumidores frente a la información adicional que muestran los productos.

Con los resultados obtenidos podemos concluir que la ley cumple con entregar información adicional para el consumidor que lo lleva a modificar su opción de compra y, contrastando con los resultados cualitativos de las encuestas revisadas en la sección 2.2, logra mejorar sus opciones de alimentación al desarrollar un mercado alimenticio más transparente con consumidores mejor informados y más preocupados por los efectos que genera una mala alimentación. 


\section{Conclusiones}

Los resultados muestran que la entrada en vigencia de la ley se relaciona con una disminución en el promedio de las unidades vendidas para la mayor parte de los productos de la muestra. Por otro lado, el número de sellos genera una variación en las ventas de cada producto, de manera significativa. En resumen, la aplicación de la ley se relaciona con una disminución de las ventas esperadas de los productos de 2 sellos, y se correlaciona con un aumento en el valor esperado de las ventas de los productos de 0,1 y 3 sellos.

En otros países la aplicación de leyes similares en el etiquetado de alimentos elaborados ha logrado resultados esperados, el consumidor ha sido capaz de tomar conciencia y castigar al producto. En Chile pareciera que la ley sí ha tenido efectos positivos en el corto plazo, sin embargo, para el caso de esta empresa y sus productos con 3 sellos podrían darse casos como que no existen productos sustitutos para modificar su consumo, que existan estrategias de posicionamiento muy efectivas que generan un alto grado de fidelización, que los consumidores de estos productos no tengan mayor disposición a cambiar de hábitos alimenticios, que durante el periodo se hayan realizado promociones adicionales para no disminuir las ventas de la empresa, entre otros. Al no tener la empresa productos con 4 sellos también queda incompleto el análisis sobre el escenario general de la introducción de la ley.

Es importante recalcar que este estudio no está exento de limitaciones. Los productos seleccionados corresponden a aquellos que tienen mayor participación en las ventas, $y$, por lo tanto, existe una variable que no ha sido incorporada en el estudio que alguna variable que estime o mida la lealtad hacia el producto. Los datos tampoco indican modificaciones en las campańas publicitarias o promociones realizadas dentro del recinto de venta junto a otro tipo de productos y sólo se consideran un tipo de canal de venta en la Región Metropolitana. Lo que deja espacio a mejorar este análisis y evaluar otros aspectos 
importantes como el comportamiento con variables como precio, género, edad, región, entre otras. Los modelos además fueron ajustados únicamente con regresiones lineales, sería interesante utilizar otras técnicas que permitan evaluar la implementación de políticas públicas y comparar sus resultados.

En términos de la efectividad de la Ley, vemos que sí ha generado cambios en la conducta del consumidor a corto plazo. Por su parte, es visible que la opinión pública se muestra a favor de legislar en el ámbito alimenticio y valora la preocupación de gobierno por mejorar los hábitos alimenticios. El etiquetado muestra ser visible para los consumidores y mejora la información en sus decisiones de consumo. La industria de alimentos procesados tiene un gran desafío gracias a esta Ley para mejorar la calidad de sus alimentos, mejorar sus ingredientes y plantear estrategia de mercadeo que no involucren a niños como parte de la estrategia de ventas. Es posible ver esta ley como una oportunidad de innovación, investigación y desarrollo para la industria de alimentos, sin generar barreras técnicas al comercio, establece límites claros para todos los participantes de la industria, y se vincula con la salud de los consumidores. Además, al estar acompañada de campañas educativas, es posible esperar que los consumidores tomen conciencia de sus hábitos alimenticios sin estigmatizar a los alimentos procesados, mostrando lo que realmente aportan a la dieta de una persona y evitando dietas desbalanceadas que generan graves problemas de salud en la población.

Para que la Ley sea más efectiva, de mejores resultados y sus efectos sean permanentes en el tiempo se debe estar constantemente informando y educando a la población. Así mismo, el gobierno debe continuamente revisar la evidencia científica sobre los efectos de los ingredientes y procesos. Del mismo modo, se recomienda ampliar la muestra de productos escogidos en este estudio e incorporar los productos sustitutos de otras marcas. Por último, se sugieren futuros estudios sobre el impacto de la ley de etiquetado de alimentos a mediano y 
largo plazo, tanto en la salud pública como en el mercado de alimentos.

\section{Notas}

${ }^{1}$ Senado. Fecha 08 de enero de 2008. Cuenta en Sesión 80, Legislatura 355 y Cámara de Diputados. Fecha 21 de enero de 2010. Cuenta en Sesión 123, Legislatura 357.

${ }^{2} \mathrm{La}$ empresa puso a disposición sus datos de ventas para este estudio con un acuerdo de confidencialidad que impide revelar su nombre.

${ }^{3}$ Estudio realizado sobre 406 encuestas efectivas. Trabajo de campo entre el 25 de julio y el 6 de agosto de 2016. Estudio exploratorio en base a metodología cuantitativa. Realizado mediante encuestas en línea a través de panel certificado ISO con más de 100.000 panelistas remunerados en Chile.

${ }^{4}$ Encuesta realizada a 3001 encuestados en 54 supermercados de la Región Metropolitana. Fecha Trabajo de Campo: Entre 25/05/16 -29/05/16. La sala EKO03-2 fue medida los días 01/05/16 y 04/05/16, y las salas JUM02-1, JUM02-2 e HIP02-1 cuentan con mediciones entre los días 07/07/16 -10/07/16. Encuestas presenciales a la salida del local, a personas que hayan comprado.

${ }^{5}$ Muestra semanal 729 casos de los cuales 531 entrevistas fueron aplicadas telefónicamente y 198 entrevistas cara a cara. Encuestas Telefónicas con Cati y encuestas Cara a Cara en puntos de afluencia con Tablet. Terrenos realizados los días Miércoles22,Jueves23yVier nes24dejuniode2016.

${ }^{6}$ Encuesta presencial en hogares, aplicada por Demoscópica. El trabajo de terreno tuvo una duración de 11 días obteniendo 1.067 casos. Estudio cuantitativo descriptivo de carácter longitudinal diseñado por el Instituto de la Comunicación e Imagen (ICEI). Cuestionario semi-estructurado, con una duración aproximada de 12 minutos, aplicada por encuestadores capacitados.

${ }^{7}$ De acuerdo a 2.147 inspecciones realizadas en supermercados y distribuidoras de alimentos,establecimientos educacionales, cines y 
elaboradoras de alimentos con y sin expendio.

${ }^{8}$ Distribución porcentual de los alimentos reportados por SOFOFA, según modificaciones en su composición nutricional. Período enero - junio 2016 con 5.434 números de casos.

${ }^{9}$ Se puso como supuesto que todos los productos bajo estudio estaban con el sello impreso al momento de partir la obligatoriedad impuesta por la ley. Sin embargo existe la posibilidad que haya un desfase las primeras semanas debido a productos que se mantenían en bodega. Sin embargo se estimó que si esta situación sucedió no afecta los resultados del estudio.

${ }^{10}$ Para esta regresión en particular se consideraron los sellos como una variable ordinal entre 0 y 3 .

BiBLIOGRAFÍA

ABADIE, A. (2005). Semiparametric difference-in-differences estimators. Review of Economic Studies, 72 (1), 1-19.

ACH. (2016, 05 01). El sobrepeso y sus efectos en la salud y vida laboral. Retrieved from Asociación Chilena de Seguridad (ACHS): http://www.achs.cl/portal/ACHS-Corporativo/ MediosACHS/Paginas/El-sobrepeso-y-sus-efectos-en-la-saludy-vida-laboral.aspx\#.WQQCMfk1-00

ALIMENTOS Y BEBIDAS DE CHILE A.G. (AB Chile). (1943). Alimentos y Bebidas de Chile A.G. (AB Chile) Misión. Retrieved 04 26, 2017, from http://abchile.cl/index. php?page=historia

Babio, N., López, L., \& Salas-Salvado, J. (2013). Análisis de la capacidad de elección de alimentos saludables por parte de los consumidores en referencia a dos modelos de etiquetado nutricional: estudio cruzado. Retrieved 05 02, 2017, from http://scielo.isciii.es/scielo.php?script=sci_arttext\&pid $=$ S0212-16112013000100024

BCN. (2011). Historia de la Ley No 20.606: Sobre composición nutricional de los alimentos y su publicidad. Santiago: Biblioteca del Congreso Nacional de Chile. 
CADEM. (2016). PLaza Pública - Track Semanal de Opinón Pública - 28 de Junio 2016. Santiago: CADEM.

CERET. (2016). Medición de la Calidad de Servicio en la Industria del Retail Supermercados. Centro de Estudios del Retail (CERET). Santiago: Departamento de Ingeniería Industrial de la Universidad de Chile.

CHAMBERS, J. M. (1992). Linear models. Capítulo 4. In J. M. Chambers, Statistical Models.

CHILE ALIMENTOS. (2008). Chile Alimentos - Quienes Somos. Retrieved 04 26, 2017, from http://www.chilealimentos.com/ wordpress/asociacion_categoria/quienes-somos/

COWBURN, G., \& STOCKLEY, L. (2005). Consumer understanding and use of nutrition labelling: a systematic review. Public health nutrition, 8 (1), 21-28.

CUADRADO, C. (2016). Medición de la carga económica actual de la obesidad en Chile y proyección al año 2030. Santiago: FONIS/CONICYT.

CUADRADO, C. (2016). The Health And Economic Burden of Obesity In Chile - An Epidemiological And Economic Simulation Model. 19 (5).

DENIS, F. (2010). Les méthodes économétriques d'évaluation. Revue française des affaires sociales (1), 105-128.

FAO. (1993). Capítulo 15 - Etiquetado nutricional. (Rome Declaration on Nutrition and the Framework of Action) Retrieved 04 26, 2017, from http://www.fao.org/docrep/ V4700S/v4700s0j.htm

FAO. (2016). Panorama de la Seguridad Alimentaria y Nutricional en América Latina. Organización de las Naciones Unidas para la Alimentación y la Agricultura (FAO) y Organización Panamericana de la Salud (OPS).

FUDIN, S. (2013, 04 02). Cost of Obesity: NPHW Infographic Winner. Retrieved from https://publichealthonline.gwu.edu/ cost-obesity-infographic-nphw/

GONZÁLEZ, S. R., ROMERO, J., TAMER, M. H., \& GUERRA, A. (2012). Un estudio del etiquetado nutricional. Culcyt , 47 
(9), 39--51.

GOOGLE. (2016). Food Trends Report. Estados Unidos: Google Trends.

GUH, D. P., ZHANG, W., BANSBACK, N., AMARSI, Z., BIRMINGHAM, C. L., \& ANIS, A. H. (2009). The incidence of co-morbidities related to obesity and overweight: A systematic review and meta-analysis. BMC Public Health, 9 (1), 1471-2458.

HENNEBERY, S., \& ARMBRUSTER, W. (2003). Emerging Roles for Food Labels: Inform, Protect, and Persuad. Journal of Food Distribution Research , 34 (3), 62-69.

IMBENS, G. W., \& WOOLDRIDGE, J. M. (2009). Recent Developments in the Econometrics of Program Evaluation. Journal of Economic Literature , 47 (1), 5-86.

INSITU. (2016). Sondeo ley de etiquetados de alimentos - Estudio

Cuantitavo . Santiago: Presentación Agosto 2016.

KATZMARZYK, P. T., JANSSEN, I., \& ARDERN, C. I. (2003). Physical inactivity, excess adiposity and premature mortality. Obes Rev , 4 (4), 257-290.

KOPELMAN, P. G. (2000). Obesity as a medical problem. Nature (404), 635-643.

KORDA, R. J., JOSHY, G., PAIGE, E., BUTLER, J. R., JORM, L. R., LIU, B., ET AL. (2015). The Relationship between Body Mass Index and Hospitalisation Rates, Days in Hospital and Costs: Findings from a Large Prospective Linked Data Study. PLOS ONE 10(3): e0118599. , 10 (3), 16.

LEIVA, M. (2014). Programa Chile Crece Sano - Presentación Gerente de Alimentos Moisés Leiva. Santiago, Chile: Chilealimentos.

LÓPEZ, M. (2017). EL Comidista - Diario El Pais. Retrieved 05 04, 2017, from http://elcomidista.elpais.com/ elcomidista/2017/04/25/articulo/1493109504_995263.html MINSAL. (2017). “Obesidad un Problema País: Evaluación de la Ley Sobre Composición Nutricional de los Alimentos y su Publicidad. Seminario Realizado por el MINSAL, 4 y 5 enero 
de 2017 . Santiago, Chile: Subsecretaría de Salud Pública. MINSAL. (2011). Encuesta Nacional de Salud. Chile 2009-2010. Santiago de Chile: Ministerio de Salud. Gobierno de Chile. MINSAL. (2017). Obesidad Un Problema País: Evaluación De La Ley Sobre Composición Nutricional De Los Alimentos Y Su Publicidad. Enero 2017 - Santiago, Chile: Ministerio de Salud. MINSAL. (1999). Reglamento Sanitario de los Alimentos. Ministerio de Salud. Santiago: Ley Chile.

MINTEL. (2017). Tendencias globales de alimentación y bebidas 2017. España: MINTEL.

TELLO, J. (2015). Brecha en los costos laborales debido a la obesidad de los trabajadores. Contaduria Universidad de Antioquia, 67, 21-44.

U.S. DEPARTMENT OF HEALTH \& HUMAN SERVICE. (2016, 08 15). Adult Obesity Causes \& Consequences. Retrieved from Centers for Disease Control and Prevention: https://www.cdc.gov/obesity/adult/causes.html

WCRF. (2009). Policy and Action for Cancer Prevention. Food, Nutrition, and Physical Activity: A Global Perspective. Washington, DC: AICR: World Cancer Research Fund/ American Institute for Cancer Research.

WHO. (2003). Diet, Nutrition and the Prevention of Chronic Diseases. Report of aJoint WHO/FAO Expert Consultation. Technical Report Series no.916. Geneva: WHO.: World Health Organization.

WILKINSON, G. N., \& ROGERS, C. E. (1973). Symbolic descriptions of factorial models for analysis of variance. Applied Statistics, 22, 392-399.

WITHROW, D., \& ALTER, D. A. (2011). The economic burden of obesity worldwide: a systematic review of the direct costs of obesity. Obesity Review , 12 (2), 131-141.

Fecha de Recepción del Artículo: 26 de marzo de 2017 Fecha de Aceptación: 06 de mayo de 2017 